\title{
Guanidine Chemistry
}

\author{
Tsutomu IsHIKAWA \\ Graduate School of Pharmaceutical Sciences, Chiba University; 1-33 Yayoi, Inage, Chiba 263-8522, Japan. \\ Received July 27, 2010
}

\begin{abstract}
Guanidines are categorized as strong organobases; however, their catalytic utility in organic synthesis has not been discussed thoroughly. The author's group has extensively and systematically studied their potential ability focusing on: 1) modified guanidines as chiral auxiliaries; 2) guanidinium ylides for aziridine formation; 3) the affinity of bisguanidine for proton and metal salts; and 4) the potential chirality of bisguanidine. Under the first topic, a variety of chiral guanidines was designed by the introduction of chirality on the three guanidinyl nitrogens, and the modified guanidines prepared using our original methods were found to be effective not only in catalytic but also in stoichiometric asymmetric syntheses. Under the second topic, the reaction of guanidinium salts carrying a glycinate function with aromatic or unsaturated aldehydes under basic conditions unexpectedly afforded aziridine-2-carboxylates, which were available as useful building blocks in organic synthesis due to their convertibility to functionalized amino acid derivatives in the ring-opening reaction, together with urea compounds recyclable to the starting guanidinium salts. The introduction of a chiral template to the guanidinium salt allowed us to expand the cyclic aziridination reaction to an asymmetric version. Under the third topic, effective complexabilty of bisguanidines with either proton or metal ions in water was observed, suggesting their possible application to the removal of toxic substances from polluted water and recovery of rare elements as material sources. Under the final topic, monomethylation or monoethylation of bisguanidine afforded a chiral product via asymmetric crystallization, indicating that bisguanidines have a potential chiral character due to the plane asymmetry.
\end{abstract}

Key words guanidine; guanidinium ylide; aziridine; bisguanidine; chiral auxiliary; base ligand

We introduced 2-chloro-1,3-dimethylimidazolinium chloride (1), which appears in 'The Merck Index' (13th ed.) as DMC (no. 3234 on p. 596), as a powerful reagent with multiple functions in synthetically useful reactions such as chlorination, oxidation, reduction, and rearrangement, in addition to the fundamental dehydration reaction under nearly neutral conditions. ${ }^{1-3)}$ On the other hand, we found that DMC (1) is easily reacted with various primary amines to give the corresponding guanidine derivatives $\mathbf{2}$, which should act as organobase catalysts for a range of asymmetric synthesis after the introduction of chirality into the template, and that either alkylation of $\mathbf{2}$ or direct amination of $\mathbf{1}$ with secondary amines provides the corresponding guanidinium salts $\mathbf{3}$, which could be transformed into previously unknown guanidinium ylides with new potential reactivity by treatment with base (Chart 1). These findings led us to study guainidine chemistry extensively and systematically. Our investigations focused on four research topics: 1) modified guanidines as chiral auxiliaries (functionality as organosuperbases); 2) guanidinium ylides for aziridine formation (role as nitrogen

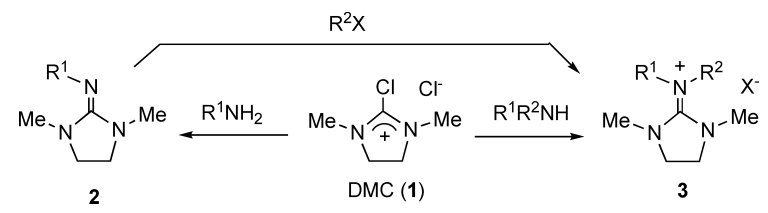

Chart 1. Reaction of DMC (1) with Amines source); 3) affinity of bisguanidine for proton and metal salts (guanidine function as a base ligand); and 4) desymmetrization of bisguanidine in alkylation (potential chirality of bisguanidine), which are discussed in this review.

\section{Modified Guanidines as Chiral Auxiliaries (Functionality as Organosuperbases)}

Guanidine is categorized as an organosuperbase with amine basicity due to the resonance stabilization of its conjugated acids; however, its catalytic utility in organic synthesis has not been discussed thoroughly. A variety of chiral guanidines could be designed by the introduction of chirality on the three guanidinyl nitrogens. It is theoretically possible to introduce five chiral centers in the guanidine system. We found that modified guanidines prepared using our original methods $^{4-6)}$ were effective not only in catalytic ${ }^{7-13)}$ but also in stoichiometric asymmetric syntheses. ${ }^{14,15)}$ The concept of modified guanidines as chiral auxiliaries in asymmetric synthesis is illustrated in Chart $2,{ }^{16-18)}$ in which reactive, but resonance-stabilized, guanidinium salts 5 could be produced from guanidines $\mathbf{4}$ by quarternarization with a reagent (A-B). The salts $\mathbf{5}$ may react with activated unsaturated substrates $(\mathrm{C}=\mathrm{D})$ to give addition products such as in the Michael reaction, trimethylsilyl (TMS) cyanation, or nucleophilic epoxidation, in which guanidines should act as catalysts (route A in Chart 2). On the other hand, the guanidinium salts 5 could serve as either an electrophile or a nucleophile. Thus, in the case of the former species, hydrogen-substituted products 
(EO-A) and protonated guanidines $(\mathbf{4} \cdot \mathrm{HB})$ may be produced (route B in Chart 2), such as the kinetic resolution of racemic sec-alcohols $(\mathrm{EOH})$ by silylation, whereas $\mathrm{OH}$-substituted products (E-B) and alternative protonated guanidines (4. HOA) could be formed in the latter cases (route $\mathrm{C}$ in Chart 2). Furthermore, according to route B in Chart 2, the electrophilic displacement of $\mathbf{5}$ with acidic compounds such as carboxylic acids may lead to the resolution of racemic secalkyl halides when alkyl halides are used for the guanidinium salt formation as A-B. In these substitution reactions, guanidines are needed in stoichiometric amounts.

Preparation of Modified Guanidines Modified guanidines were prepared using four synthetic methodologies based on the versatile character of DMC (1): reaction of DMC (1)-like chloroamidine intermediates with primary amines (method I); the DMC (1)-induced cyclization of protected thioureas (method II); intramolecular $S_{N} 2$ reactions of hydroxyethyl-substituted guanidines after chlorination with DMC (1) (method III); and a combination of the DMC (1)induced cyclization of thioureas and intramolecular $S_{N} 2$ reaction (method IV).

Using method I, 1,3-disubstituted 2-iminoimidazolidines 6 and bicyclic guanidines 7 were prepared as shown in Chart 3. ${ }^{4)}$

Method II allows the preparation of 1- and/or 3-unsubstituted 2-iminoimidazolidines derived from the ethylenedi-

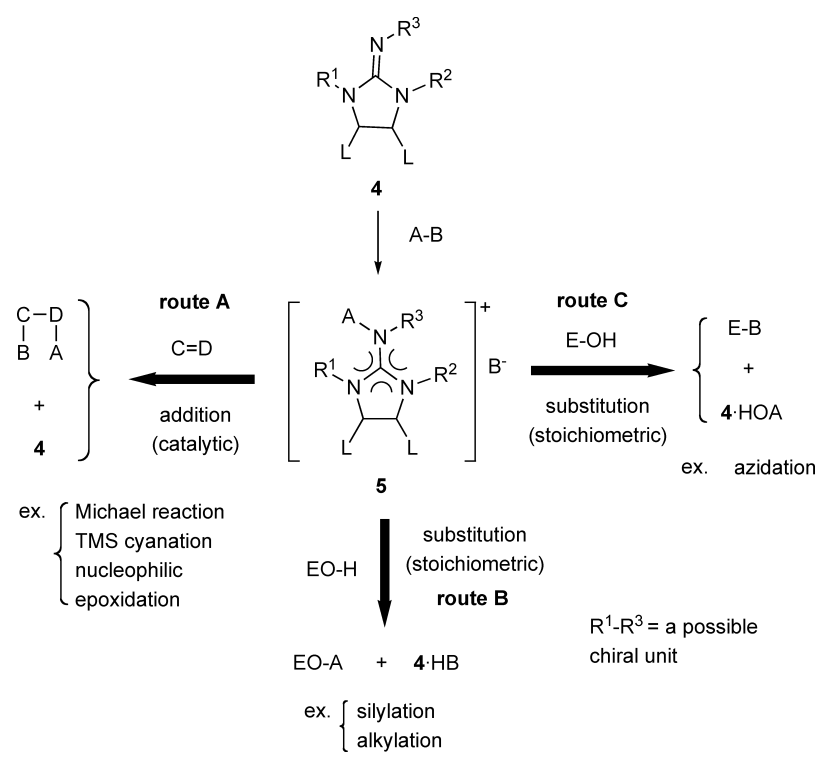

Chart 2. Concept of Modified Guanidines as Chiral Auxialiaries amines (Chart 4). ${ }^{5)}$ Thus 2-aminoimidazolidines 9 were prepared from trisubstituted thioureas $\left(8: R^{1}, R^{2} \neq H\right)$, and 1,3unsubstituted $\mathbf{1 0}$ and 1-substituted 2-iminoimidazolidines $\mathbf{1 1}$ from disubstituted thioureas $\left(\mathbf{8}: \mathrm{R}^{2}=\mathrm{H}\right)$ through protected imidazolidine derivatives.

The treatment of hydroxyethyl-substituted guanidines with DMC (1) afforded alternative guanidines using method III (Chart 5). ${ }^{6}$ Thus 3,7,8-trisubstituted 1,4,6-triazabicyclooctenes 13 and 1,4-disubstituted 2-iminoimidazolidines 15 were prepared from 1-unsubstituted 2-(2-hydroxyethylimino)imidazolidines $\mathbf{1 2}$ and linear-type guanidines $\mathbf{1 4}$ through chlorine-displaced intermediates, respectively. Double DMC (1)-induced cyclization of guanidines 16 containing two 2hydroxyethyl substituents provided another type of 1,4,6-triazabicyclooctene system 17.

DMC (1)-induced cyclization of thiourea 18 followed by the intramolecular $S_{N} 2$ reaction of the monocyclic guanidine 19 formed gave a $C_{2}$-symmetrical bicyclic guanidine 20 using method IV (Chart 6). ${ }^{9)}$

Application to Asymmetric Synthesis Michael reac-
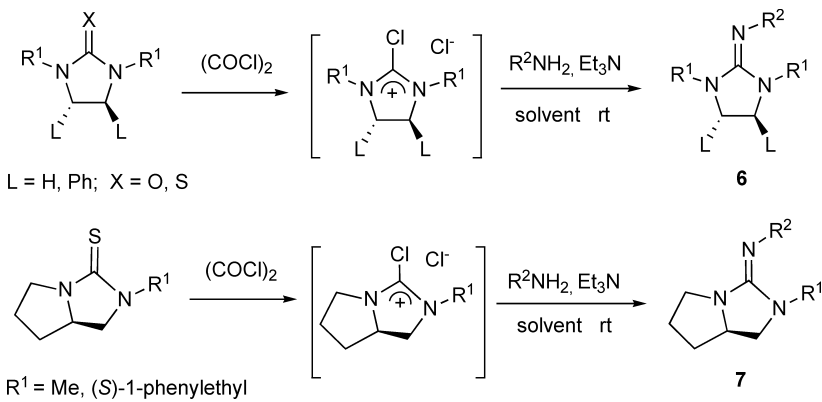

Chart 3. Preparation of Guanidines Using Method I
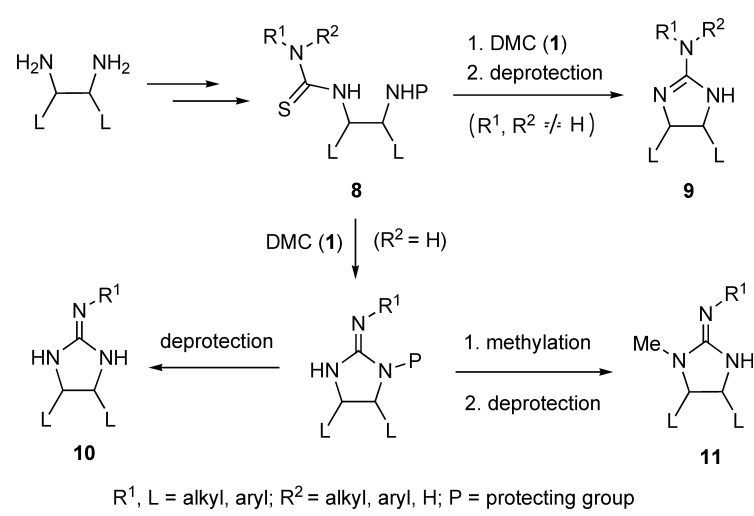

Chart 4. Preparation of Guanidines Using Method II

Tsutomu Ishikawa was born in 1949. He studied organic chemistry at Faculty of Pharmaceutical Sciences, Chiba University, took a position at the same university as a research associate after graduation, and was promoted to associate professor in 1992 and then to full professor in 1996. During his career, he received a PhD from Kyoto University in 1980 and worked at Dyson Perrins Laboratory, Oxford University, U.K., as a postdoctoral fellow in Dr. Laurence M. Harwood's group from 1989 to 1990. His research interests are medicinal organic chemistry, including the total synthesis and isolation of bioactive natural products, and basic organic chemistry focusing on environment-friendly functioning materials.

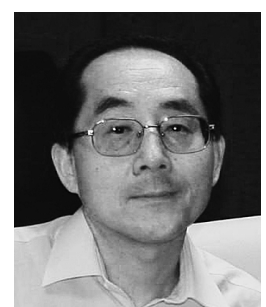

Tsutomu IsHIKAWA 
tions between diphenyliminoacetate $\mathbf{2 1}$ and vinyl compounds 22 are examples of guanidine-catalyzed asymmetric synthesis (Table 1). ${ }^{7,11)}$ A 1,3-disubstituted $(4 S, 5 S)$-diarylimidazolidine skeleton 23 with an (R)-1-(2-hydroxymethyl)-2-phenylethylimino moiety at the 2 position (or the enantiomer), which can be prepared using method I, was found to be the most effective catalyst in these reactions, in which an $(R)$-excess adduct $\mathbf{2 4}$ was obtained. Treatment with ethyl acrylate (22a) as a vinyl compound in the presence of catalyst 23a [designated as " $(+)$-Chiba-G"] smoothly afforded the adduct 24a (97\% ee in $85 \%$ yield) when the reaction was carried out
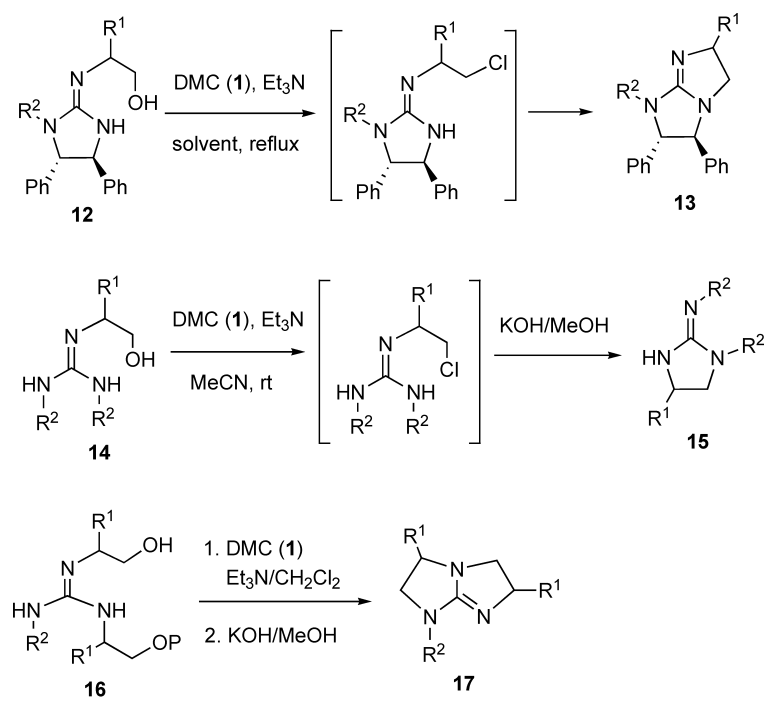

$\mathrm{R}^{1}=$ alkyl, aryl; $\mathrm{R}^{2}=$ alkyl, aryl, protecting goup, $\mathrm{H}$ (after deprotection)

Chart 5. Preparation of Guanidines Using Method III

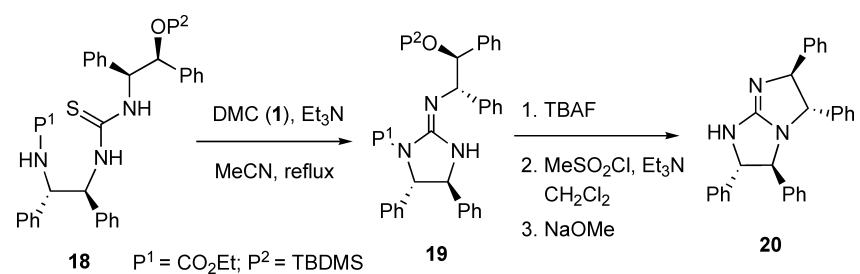

Chart 6. Preparation of Guanidine Using Method IV without solvent (for $3 \mathrm{~d}$ ), whereas a less effective reaction ( $79 \%$ ee in $15 \%$ yield) was observed in tetrahydrofuran (THF) solution (for $7 \mathrm{~d}$ ). ${ }^{7)}$ Interestingly, replacement of the acrylate 22a with methyl vinyl ketone (22b) led to higher asymmetric induction in THF (96\% ee in $90 \%$ yield) than without solvent ( $80 \%$ ee in $90 \%$ yield). In general, remarkable rate acceleration was observed in solventless reactions. Either displacement of the 4,5-diphenyl units in Chiba-G 23a to 2-tolyl ones $\mathbf{2 3} \mathbf{b}$ or modification of the 1,3-dimethyl substituents in Chiba-G 23a to the bulkier benzyl ones 23c led to the improvement of asymmetric induction, albeit the reactivity was lowered in the case of the latter $\mathbf{2 3 c} .^{11)}$

We assumed the formation of a relatively tight complex through not only a hydrogen bond but also the $\mathrm{CH}-\pi$ interaction in the transition state leading to effective enantioselectivity in the adduct 24, as shown in Fig. 1. ${ }^{11)}$

The (+)-Chiba-G (23a)-catalyzed Michael reaction of diphenyliminoacetate $\mathbf{2 1}$ was applied to the enantioselective construction of nicotine skeleton 27 using pyridyl vinyl ketone (26), derived from a vinyl alcohol 25, as a Michael acceptor. ${ }^{13)}$ Satisfactory results were obtained, as shown in Chart 7.

Kinetic resolution of $\mathrm{sec}$-alcohols by silylation is an example of a stoichiometric reaction. ${ }^{15)}( \pm)$-Indanol (28) was treated with tert-butyldimethylsilyl chloride (TBDMSCl) in the presence of a variety of modified guanidines (Chart 8). 1,3-Unsubstituted guanidine 30, its 1-methyl derivative 31, and (3S,7S,8S)-3-benzyl-7,8-diphenyl-1,4,6-triazabicy-

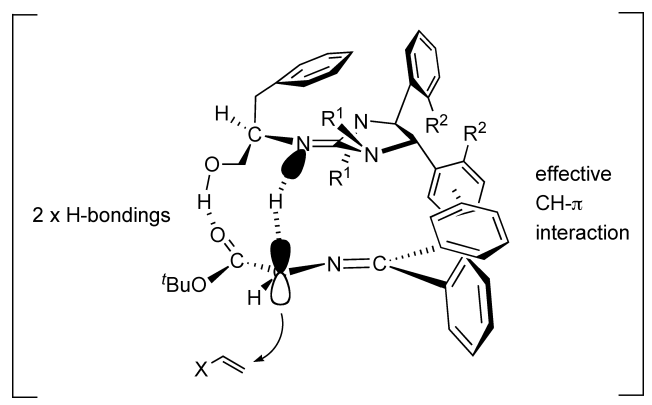

Fig. 1. Assumed Transition State for the Guanidine-Catalyzed Michael Reaction

Table 1. Example of Guanidine-Catalyzed Michael Reaction

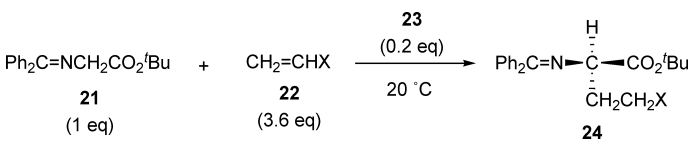

\begin{tabular}{|c|c|c|c|}
\hline 23 & $22(X)$ & In THF/time ${ }^{a)}$ & Without solvent/time ${ }^{a)}$ \\
\hline \multirow{3}{*}{$\begin{array}{l}\text { a: } \mathrm{R}^{1}=\mathrm{Me} \\
\mathrm{R}^{2}=\mathrm{H} \\
{[(+) \text {-Chiba-G }]}\end{array}$} & a: $\mathrm{CO}_{2} \mathrm{Et}$ & $\begin{array}{l}15 \% / 7 \mathrm{~d} \\
(79 \% \text { ee })\end{array}$ & \multirow{5}{*}{$\begin{array}{l}85 \%(100 \%) / 3 \mathrm{~d} \\
(97 \% \text { ee }) \\
90 \%(100 \%) / 15 \mathrm{~h} \\
(80 \% \text { ee }) \\
79 \%(100 \%) / 5 \mathrm{~d} \\
(55 \% \text { ee })\end{array}$} \\
\hline & b: $\mathrm{COMe}$ & $\begin{array}{l}90 \% / 6 \mathrm{~d} \\
(96 \% \text { ee })\end{array}$ & \\
\hline & c: $\mathrm{CN}$ & NR & \\
\hline $\begin{aligned} \text { b: }: \mathrm{R}^{1} & =\mathrm{Me} \\
\mathrm{R}^{2} & =\mathrm{Me}\end{aligned}$ & a: $\mathrm{CO}_{2} \mathrm{Et}$ & $\begin{array}{l}62 \%(100 \%) / 7 \mathrm{~d} \\
(90 \% \text { ee })\end{array}$ & \\
\hline c: $\begin{aligned} \mathrm{R}^{1} & =\mathrm{CH}_{2} \mathrm{Ph} \\
\mathrm{R}^{2} & =\mathrm{H}\end{aligned}$ & a: $\mathrm{CO}_{2} \mathrm{Et}$ & $\begin{array}{l}27 \% / 7 \mathrm{~d} \\
(98 \% \text { ee })\end{array}$ & \\
\hline
\end{tabular}

a) Isolated, non-optimized yield. Parenthesis shows the estimation by ${ }^{1} \mathrm{H}-\mathrm{NMR}$ spectrum. 
clo[3.3.0]-oct-4-ene (32) were found to be effective auxiliaries. A higher $(\mathrm{ca} .70 \%)$ ee value was observed when a bulkier triisopropylsilyl chloride was used as a silylating agent. This was the first example of the enantioselective silylation of alcohol.

\section{Guanidinium Ylides for Aziridine Formation (Role as Ni- trogen Source)}

The application of guanidinium ylides to organic synthesis was not previously reported in the literature to the best of our knowledge; however, these would be expected to act as stabilized equivalents of azomethine ylides due to their resonance stabilization. In the course of our studies of guanidine chemistry, we found that the treatment of guanidinium salts 33 containing a glycine unit with aryl aldehydes in the presence of a base directly afforded 3-arylaziridine-2-carboxylates 35 in high yields with excellent to moderate diastereoselectivity and that the introduction of chiral centers into the guanidinium template $(33: \mathrm{L}=\mathrm{Ph})$ resulted in effective asymmetric induction of aziridine formation (Chart 9). ${ }^{19)}$ In the reaction (Chart 9), the 1,3-dimethylimidazolidin-2-one (DMI) derivative 36 is co-produced as a reusable source for the guanidinium template $\mathbf{3 3}$, and thus this unique aziridination reaction could be formally categorized as an atom-economical cycle reaction because there is no waste of any of the key components during reactions.

Aziridines from Guanidinium Ylides and Aryl Aldehydes The reaction of guanidinium bromide $\mathbf{3 7}$ with an aryl aldehyde in the presence of either sodium hydride $(\mathrm{NaH})$ in
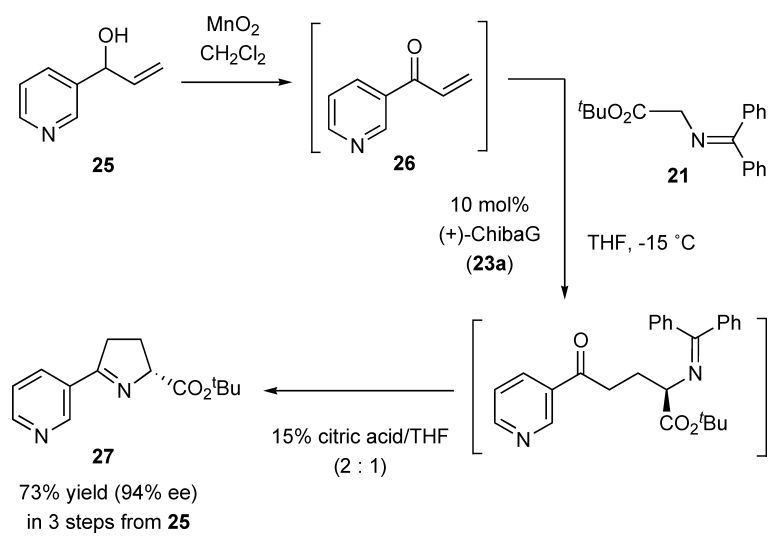

Chart 7. Application of the (+)-Chiba-G (23a)-Catalyzed Michael Reaction for the Construction of Nicotine Skeleton 27

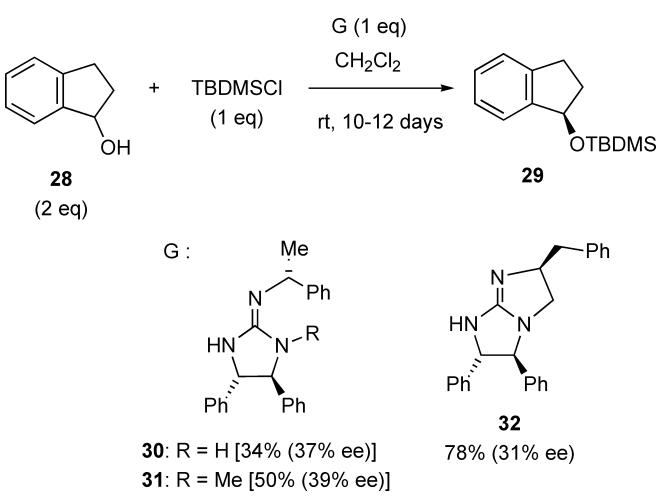

Chart 8. Guanidine-Induced Kinetic Resolution of Indanol (28) dimethylformamide (DMF) or tetramethylguanidine (TMG), followed by treatment with silica gel $\left(\mathrm{SiO}_{2}\right)$, afforded 3-arylaziridine-2-carboxylate $\mathbf{3 8}$ in reasonable yield together with DMI (39) (Table 2). ${ }^{19)}$ This synthetic method is widely applicable to a variety of aryl aldehydes including heterocycles, in which trans-aziridines trans-38 were preferentially formed. In particular, not only trans selectivity ( $c$. 90\% de) but also satisfactory conversion (68-95\%) were observed when electron-rich benzaldehydes (runs 3-6) and indolyl aldehydes (runs 12 and 13) were used. Cinnamaldehyde could also be converted to 1,2-addition-type aziridine product $\mathbf{3 8 g}$ in $70 \%$ yield (run 11).

Extension to Asymmetric Aziridination This aziridine synthesis is applicable to asymmetric synthesis (Table 3). ${ }^{19)}$ A smooth reaction was observed when the chiral guanidinium bromide $\mathbf{4 0}$ was treated with aryl aldehydes under the same conditions as achiral salts, in which TMG (run 3) is more effective in both chemical yield and stereoselectivity than $\mathrm{NaH}$ in DMF (run 4).

The absolute stereochemistry of aziridine products was determined by chemical correlation of 3-phenylaziridine-2-carboxylates (cis-41a: 79\% ee; trans-41a: 77\% ee) obtained in run 1 in Table 3 with commercially available $(S)$-phenylalaninate 44, as shown in Chart $10 .{ }^{19)}$ Each hydrogenolysis of the isomeric aziridines 41a afforded the same $N$-protected phenylalaninate $\mathbf{4 3}$, but its absolute configuration was opposite to that of the product $\mathbf{4 3}$ derived from $(S)$-phenylalaninate 44 . Thus it was established that the $(S, S)$-guanidinium ylide $(S, S)-\mathbf{4 0}$ mainly produces cis-(2R,3R)- and trans$(2 R, 3 S)$-3-arylaziridine-2-carboxylates, strongly suggesting that the $\mathrm{C}-2$ stereogenic centers in the cis- and trans-aziridine skeletons are controlled in the same fashion. In other words, it is $\mathrm{C}-\mathrm{C}$ bond formation, but not $\mathrm{N}-\mathrm{C}$ bond formation, that should play a crucial role in the enantioselectivity in this reaction between guanidinium ylide and aryl aldehyde.

Unsaturated aldehydes including acetylenic ones also served as electrophiles in this asymmetric aziridination reac-

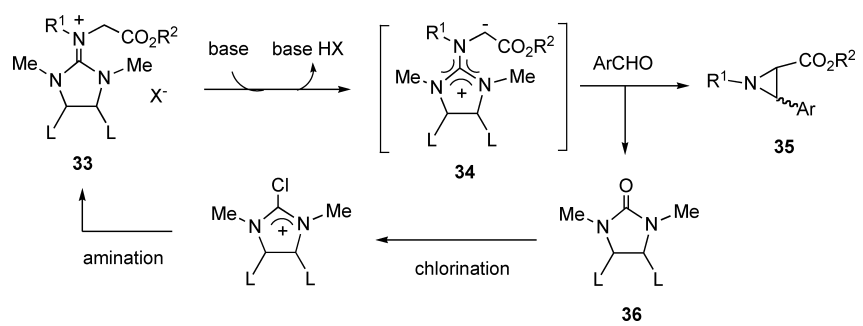

Chart 9. Aziridine Formation by Reaction of Guanidinium Salt with Aryl Aldehyde in the Presence of Base

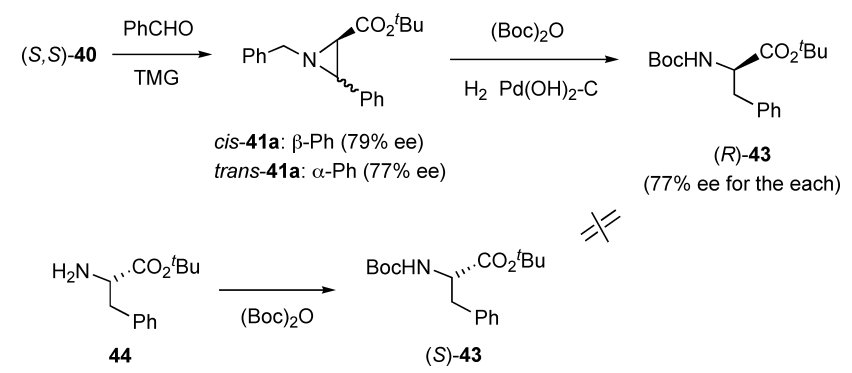

Chart 10. Determination of the Absolute Stereochemistry of 3-Phenylaziridine-2-carboxylates 41a 
Table 2. Reaction of Guanidinium Salt with Aryl Aldehydes

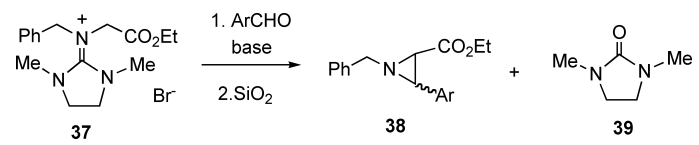

Ar: $a=P h, \quad b=3,4-\left(\mathrm{OCH}_{2} \mathrm{O}\right) \mathrm{C}_{6} \mathrm{H}_{4}, \quad \mathrm{c}=4-\mathrm{MeOC}_{6} \mathrm{H}_{5}, \quad \mathrm{~d}=2-\mathrm{MeOC}_{6} \mathrm{H}_{5}$, $\mathrm{e}=4-\mathrm{ClC}_{6} \mathrm{H}_{5}, \mathrm{f}=4-\mathrm{NO}_{2} \mathrm{C}_{6} \mathrm{H}_{5}, \mathrm{~g}=\mathrm{PhCH}=\mathrm{CH}, \mathrm{h}=3-[(1-\mathrm{Boc})$ indolyl $], \mathrm{i}=2$ [(1-Boc)indolyl], $\mathrm{j}=3$-Pyridyl

\begin{tabular}{|c|c|c|c|c|c|c|c|c|}
\hline \multirow{2}{*}{ Run } & \multirow{2}{*}{$\mathrm{Ar}$} & \multirow{2}{*}{ Base $^{a)}$} & \multirow{2}{*}{$\begin{array}{l}\text { Time } \\
\text { (h) }\end{array}$} & \multicolumn{4}{|c|}{ Yield $(\%)^{b)}$ of $\mathbf{3 8}$} & \multirow{2}{*}{$\begin{array}{c}39 \\
(\%)\end{array}$} \\
\hline & & & & cis & trans & cis/trans & Total & \\
\hline 1) & $\mathrm{a}$ & $\{\mathrm{A}$ & 24 & $\{23$ & 61 & $27 / 73$ & 84 & 78 \\
\hline 2$\}$ & & $\left\{\begin{array}{l}\mathrm{B} \\
\mathrm{B}\end{array}\right.$ & 24 & $\left\{\begin{array}{l}28 \\
28\end{array}\right.$ & 41 & $41 / 59$ & 69 & 77 \\
\hline 3) & $\mathrm{b}$ & $\int \mathrm{A}$ & 24 & $\int 8$ & 69 & $10 / 90$ & 77 & 71 \\
\hline 4$\}$ & & $\{\mathrm{B}$ & 24 & 4 & 64 & $6 / 94$ & 68 & 83 \\
\hline 5 & $\mathrm{c}$ & A & 9 & 7 & 78 & $8 / 92$ & 85 & -c) \\
\hline 6 & $\mathrm{~d}$ & A & 8 & 2 & $93^{(d)}$ & $2 / 98$ & 95 & -c) \\
\hline 7) & & A & 24 & $\{17$ & 48 & $26 / 74$ & 65 & 93 \\
\hline 8$\}$ & $\mathrm{e}$ & $\left\{\begin{array}{l}\text { B } \\
\text { B }\end{array}\right.$ & 24 & $\{16$ & 45 & $26 / 74$ & 61 & 94 \\
\hline 9$\}$ & & $\int \mathrm{A}$ & 24 & $\int 2$ & 45 & $4 / 96$ & 47 & -c) \\
\hline 10$\}$ & $\mathrm{f}$ & $\left\{\begin{array}{l}\mathrm{B} \\
\mathrm{B}\end{array}\right.$ & 2 & 3 & 43 & $7 / 93$ & 46 & 72 \\
\hline 11 & $\mathrm{~g}$ & A & 24 & 17 & $53^{d)}$ & $24 / 76$ & 70 & -c) \\
\hline 12 & $\mathrm{~h}$ & A & 24 & 4 & $72^{d)}$ & $5 / 95$ & 76 & 88 \\
\hline 13 & $\mathrm{i}$ & A & 24 & 4 & $75^{d)}$ & $5 / 95$ & 79 & 88 \\
\hline 14 & $\mathrm{j}$ & A & 7 & 8 & 58 & $12 / 88$ & 66 & 72 \\
\hline
\end{tabular}

a) A: NaH. The reaction was carried out in $\mathrm{DMF}$ at $-20^{\circ} \mathrm{C}$. B: TMG. The reaction was carried out without solvent at rt. b) Isolated, non-optimized yield. c) Isolation was not attempted. d) A mixture of two invertomers at the nitrogen atom.

Table 3. Selected Examples of Asymmetric Aziridine Synthesis

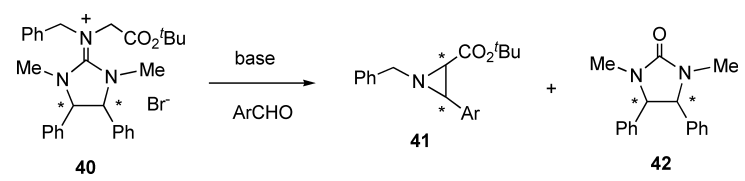

\begin{tabular}{|c|c|c|c|c|c|c|c|c|c|}
\hline \multirow{2}{*}{ Runs $^{a)}$} & \multirow{2}{*}{40} & \multirow{2}{*}{$\mathrm{Ar}$} & \multirow{2}{*}{ Base } & \multirow{2}{*}{$\begin{array}{l}\text { Time } \\
\text { (h) }\end{array}$} & \multicolumn{4}{|c|}{ Yield $^{b}(\%)$ of $\mathbf{4 1}^{c}$} & \multirow{2}{*}{$\begin{array}{c}42 \\
(\%)\end{array}$} \\
\hline & & & & & cis $(\mathrm{ee} \%)$ & trans $(\mathrm{ee} \%)$ & cis/trans & Total & \\
\hline 1 & $(S, S)$ & $\mathbf{a}$ & B & 3 & $60(79)$ & $31(77)$ & $66 / 34$ & 91 & 88 \\
\hline 2 & $(R, R)$ & a & B & 5 & $61(75)$ & $32(73)$ & $66 / 34$ & 93 & 96 \\
\hline 3 & $(S, S)$ & b & B & 4 & $6(-)$ & $82(97)$ & $7 / 93$ & 88 & 91 \\
\hline 4 & $(R, R)$ & b & A & 39 & $7(-)$ & $75(72)$ & $9 / 91$ & 82 & 86 \\
\hline 5 & $(S, S)$ & e & B & 4 & $51(-)$ & $35(59)$ & $59 / 41$ & 86 & $-d)$ \\
\hline 6 & $(S, S)$ & h & B & 7 & $6(-)$ & $70(95)$ & $8 / 92$ & 76 & 87 \\
\hline 7 & $(S, S)$ & i & B & 5.5 & $9(-)$ & $87(76)$ & 9/91 & 96 & 90 \\
\hline
\end{tabular}

a) The reaction was carried out using the same ArCHO under the conditions shown in Table 2. b) Isolated, non-optimized yield. c) ee was determined by chiral HPLC. d) Isolation was not attempted.

Table 4. Selected Examples of Asymmetric Unsaturated Aziridinations

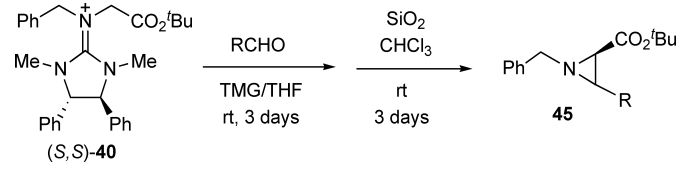

\begin{tabular}{|c|c|c|c|c|c|c|}
\hline \multirow{2}{*}{ Run } & & \multirow{2}{*}{$\mathrm{R}$} & \multicolumn{4}{|c|}{ Yield $^{a)}(\%)$ of $\mathbf{4 5}^{b)}$} \\
\hline & & & cis (ee) & trans (ee) & cis/trans & Total \\
\hline 1 & $\mathbf{a}$ & $\mathrm{CH}_{2}=\mathrm{CH}-$ & $31(60)$ & 31 & $50 / 50$ & 62 \\
\hline 2 & b & $(E)-\mathrm{C}_{13} \mathrm{H}_{27} \mathrm{CH}=\mathrm{CH}-$ & $46(91)$ & $41(96)$ & $53 / 47$ & 87 \\
\hline 3 & c & Cyclohexenyl & $33(97)$ & 18 & $65 / 35$ & 51 \\
\hline 4 & d & (E) $-\mathrm{PhCH}=\mathrm{CH}-$ & $22(67)$ & $60(74)$ & $27 / 73$ & 82 \\
\hline 5 & e & $(E)-\mathrm{MeCH}=\mathrm{C}(\mathrm{Ph})-$ & $32(91)$ & $10(89)$ & $76 / 24$ & 42 \\
\hline 6 & f & $\mathrm{PhC} \equiv \mathrm{C}-$ & $22(92)$ & $48(98)$ & $41 / 59$ & 70 \\
\hline 7 & g & $\mathrm{C}_{13} \mathrm{H}_{27} \mathrm{C} \equiv \mathrm{C}-$ & 18 & 26 & $86 / 14$ & 44 \\
\hline
\end{tabular}

a) Isolated, non-optimized yield. $\quad$ ) ) ee was determined by drival HPLC. tion. ${ }^{20)}$ Selected examples of the preparation of unsaturated aziridine-2-carboxylates $\mathbf{4 5}$ are shown in Table 4.

Mechanistic Approaches to the Aziridination Reactions Next, we systematically examined the reaction of guanidinium salt $\mathbf{4 0}$ with a variety of para-substituted benzaldehydes for mechanistic approaches. ${ }^{21)}$ A mixture of $(S, S)-\mathbf{4 0}$ and a benzaldehyde was stirred in THF in the presence of TMG at $25^{\circ} \mathrm{C}$ for $5 \mathrm{~h}$ (step 1) and then treated with acetic anhydride $\left(\mathrm{Ac}_{2} \mathrm{O}\right)$ in place of $\mathrm{SiO}_{2}$ at room temperature until disappearance of the polar intermediate on thin-layer chromatography (step 2). The aziridination reactions could be classified into four groups based on the nature of benzaldehydes used: 1) group A, which includes benzaldehydes with strong electron-donating group (EDG) $\left(\mathrm{X}=\mathrm{O}^{n} \mathrm{Bu}\right.$ and $\left.\mathrm{OMe}\right)$, is characterized by a very slow step 1 and a very rapid step 2 , showing excellent diastereoselectivity and enantioselectivity 
of trans-aziridine; 2) group B, represented by a slow step 1 and a rapid step 2 with moderate cis diastereoselectivity and excellent to good enantioselectivity in both isomeric aziridines, and containing benzaldehydes that possess a weak EDG $(X=M e)$ or no substituents $(X=H)$; 3) group $C$, showing a profile similar to group $\mathrm{B}$ but with a rapid step 1 and a slow step 2, and comprising benzaldehydes with a weak electron-withdrawing group (EWG) $\left(\mathrm{X}=\mathrm{Cl}\right.$ and $\left.\mathrm{CO}_{2} \mathrm{Me}\right)$; and 4) group $\mathrm{D}$, which includes benzaldehydes with a strong EWG $\left(\mathrm{X}=\mathrm{CN}\right.$ and $\left.\mathrm{NO}_{2}\right)$, characterized by a moderate trans diastereoselectivity and low enantioselectivity with a very rapid step 1 and a very slow step 2 . Based on these experimental results, we hypothesized the mechanistic pathway for each group, among which the mechanisms for groups A and $\mathrm{C}$ are summarized in Chart 11.

An aldehyde in group A approaches the less hindered reface $[\mathrm{re}(\mathrm{G})]$ of the ylide enolate $\mathbf{4 6}$ derived from the salt $(S, S)$-40 to induce $R$ stereochemistry of the stereogenic center in the $\mathrm{C}-\mathrm{C}$ bond formation. The less hindered re-face $[\mathrm{re}(\mathrm{A})]$ of the aldehyde participates in the bond formation, resulting in the production of a stable $(4 R, 5 S)$-oxazolidine 47 with a trans configuration between the ester and aryl groups. Then, very rapid concerted fragmentation like the $S_{N i \text {-type }}$ reaction can occur with retention at the $\mathrm{C}-5$ benzylic carbon to give trans- $(2 R, 3 S)$-aziridine trans- $(2 R, 3 S)-41$. It is reasonable to expect that $\mathrm{Ac}_{2} \mathrm{O}$ could coordinate the oxygen atom of the oxazolidine moiety (see, 48 ) and the partially cationic character of the oxygen atom in the coordinated species initiates the fragmentation of the intermediate adduct 47 to trans41 and a 2-acetoxyamidinium salt, convertible to urea $\mathbf{4 2}$, by simultaneous reactions of the $\mathrm{O}-\mathrm{C}(\mathrm{Ar})$ bond cleavage and the $\mathrm{N}$ (oxazolidine)-C(Ar) bond formation. In this reaction sequence, partially-generated benzyl cationic species could be stabilized by electron-relayed participation of the $p$-substituted alkoxy group.

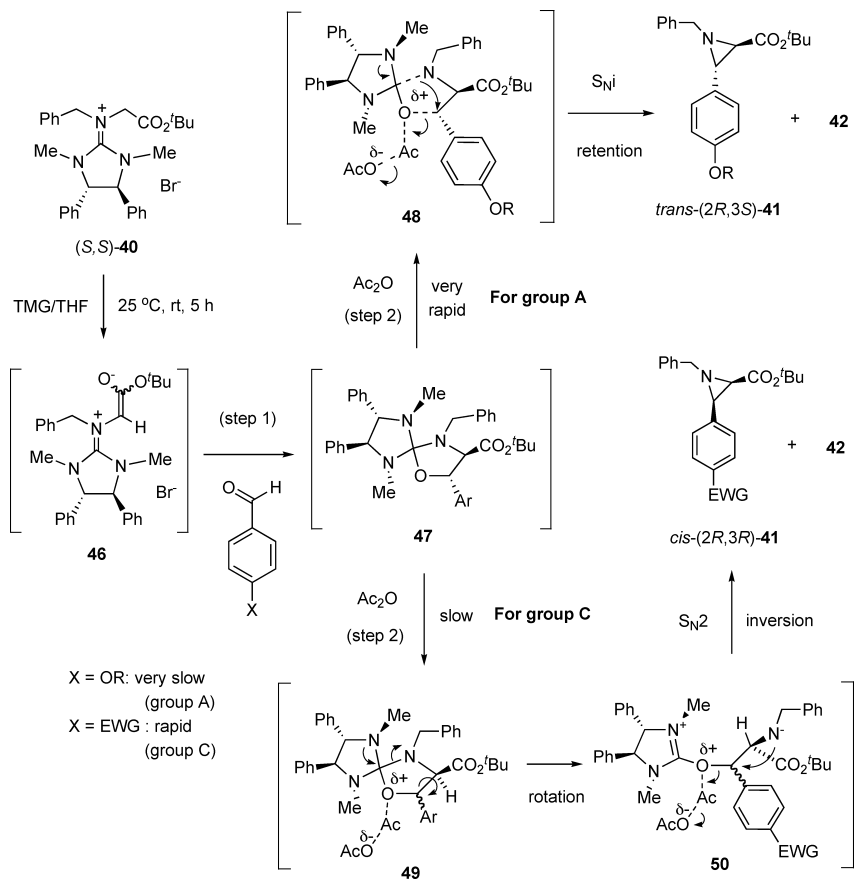

Chart 11. Assumed Main Mechanistic Pathways for Asymmetric Aziridination with Aldehydes Belonging to Groups A and C
Group $\mathrm{C}$ also allows the $r e(\mathrm{G})-r e(\mathrm{~A})$ approach as a major path in step 1 , even with an alternative $\operatorname{re}(\mathrm{G})-\operatorname{si}(\mathrm{A})$ because of the lowered face selectivity. The oxygen atom of the oxazolidine unit in the spiro intermediates $\mathbf{4 7}$ is similarly activated with $\mathrm{Ac}_{2} \mathrm{O}$ (see, 49); however, the benzyl cationic species could not be formed due to destabilization in the presence of EWG. Therefore the cleavage of the $\mathrm{C}$ (spiro)$\mathrm{N}$ (oxazolidine) bond involving the nitrogen atom of the imidazolidine unit affords an opened amidinium intermediate 50, in which an intramolecular $S_{N} 2$-type reaction occurs, resulting in the predominant production of cis- $(2 R, 3 R)$-aziridine $c i s-(2 R, 3 R)-41$ with inversion of the configuration at $\mathrm{C}$ 5 . The slightly lowered ee value of aziridines could be explained by competitively enantiofacial accesses, such as the si(G)-re(A) approach, in the rapid reaction of step 1.

These mechanistic considerations were strongly supported by the successful isolation and complete characterization using X-ray analysis of the spiro intermediate 51, which could be smoothly converted to aziridine products $\mathbf{5 2}$ by treatment with $\mathrm{SiO}_{2}$ when 2-bromocinnamaldehyde was used as an electrophile (Chart 12). ${ }^{22)}$

Reaction of phosphorus ylides with carbonyl compounds is well established as the Wittig reaction, in which olefinic products are produced by $\mathrm{C}-\mathrm{C}$ bond formation, followed by the elimination of the oxygen atom of carbonyl substrates and the phosphorus unit of ylides in oxaphosphetane intermediates as phosphine oxides (a in Chart 13). It is also known that oxirane products incorporating the oxygen atom of carbonyl compounds by intramolecular displacement of
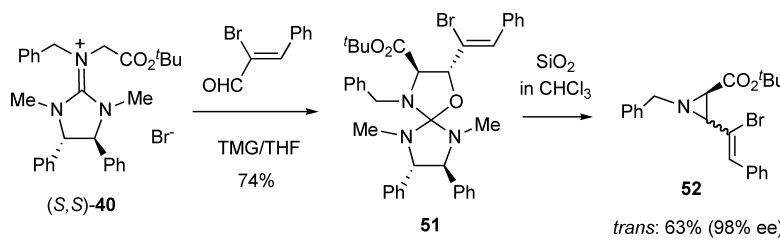

characterized by $\mathrm{X}$-ray cis: $12 \%(94 \%$ ee)

Chart 12. Isolation of a Spiro Intermediate Derived from 2-Bromocinnamaldehyde

(a) Phosphorus ylides: olefination (Wittig reaction)

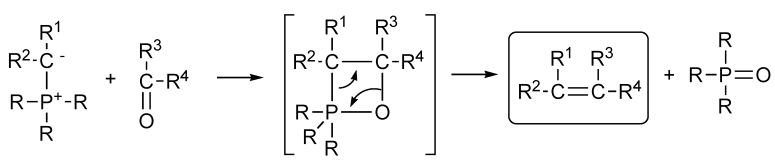

(b) Sulfur ylides: epoxidation

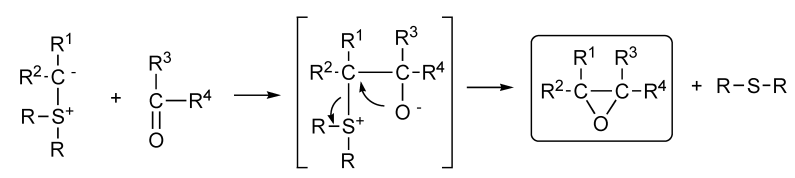

(c) Guanidinium (nitrogen) ylides: aziridination

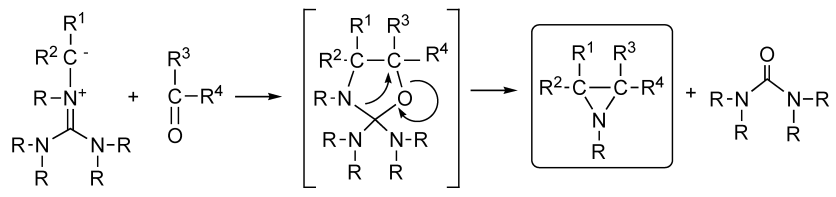

Chart 13. Comparison of the Reactions of Carbonyl Compounds with Phosphorus, Sulfur, and Guanidinium Ylides 
the sulfur atom with oxygen atom in the intermediate zwitter ions after $\mathrm{C}-\mathrm{C}$ bond formation are produced in the reaction of sulfur ylides and carbonyl compounds (b in Chart 13). On the other hand, in the guanidinium ylide-participating reactions with carbonyl compounds, aziridine products incorporating the nitrogen unit of the guanidinium ylides are produced together with urea derivatives by the fragmentation of oxazolidine intermediates (c in Chart 13). Thus our findings on guanidinium ylides can offer a remarkable contrast in product profiles from known phosphorus and sulfur ylide chemistry.

Ring-Opening Reactions of Aziridine-2-carboxylates Aziridine-2-carboxylates are very versatile synthetic intermediates for the preparation of biologically active nitrogencontaining compounds because they are convertible to $\alpha$ - or $\beta$-amino acid derivatives, including unnatural amino acids, by regioselective ring-opening reactions. Aziridines are classified into two groups, "activated" and "unactivated (or nonactivated)" aziridines, depending upon the substituent on the ring $\mathrm{N}$-atom; the former category includes electron-withdrawing substituents such as tosyl or acyl functions, whereas hydrogen and alkyl substituents are typical for the latter one. Although the reactivity of activated aziridines has been investigated extensively, there are only limited reports on unactivated aziridines. We turned our attention to the ring-opening reaction of unactivated aziridine-2-carboxylates obtained in this unique aziridination reaction for the intensive exploration of their synthetic utility.

Enantioselective total synthesis of sphingosine was targeted as the example of hetero atom-participating ring-opening reactions of unsaturated aziridine-2-carboxylates. ${ }^{20)}$ The trans- $(2 R, 3 S)$-3-pentadecenylaziridine-2-carboxylate trans$(2 R, 3 S)-\mathbf{4 5} \mathbf{b}$, obtained in run 2 in Table 4 , was treated with acetic acid to give $\alpha$-amino acetate $\mathbf{5 3}$ with inversion at the 3 -position. After hydrolysis, protection of the alcoholic function and reduction of the ester group afforded $\alpha$-amino alcohol 56. Protection of the bifunctional group in $\mathbf{5 6}$ with carbonyl diimidazole (CDI), followed by silyl deprotection and alkaline hydrolysis, provided the desired D-erythro-sphingosine (59), which was characterized by transformation to acetate 60, in good overall yield, as shown in Chart 14. The isomeric cis- $(2 R, 3 R)$-aziridine-2-carboxylate cis- $(2 R, 3 R)-\mathbf{4 5} \mathbf{b}$,

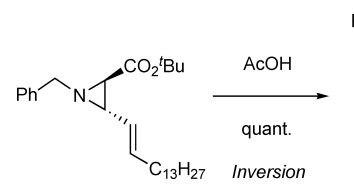

trans-(2R,3S)-45b

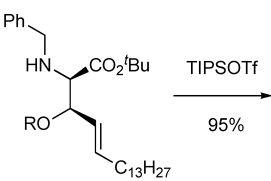

53: $\mathrm{R}=\mathrm{Ac} \rightarrow \mathrm{KOH}$

$$
\begin{aligned}
& \text { 53: } \mathrm{R}=\mathrm{Ac} \\
& \text { 54: } \mathrm{R}=\mathrm{H}
\end{aligned} \begin{aligned}
& \mathrm{KOH} \\
& 99 \%
\end{aligned}
$$

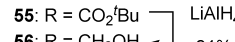

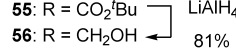

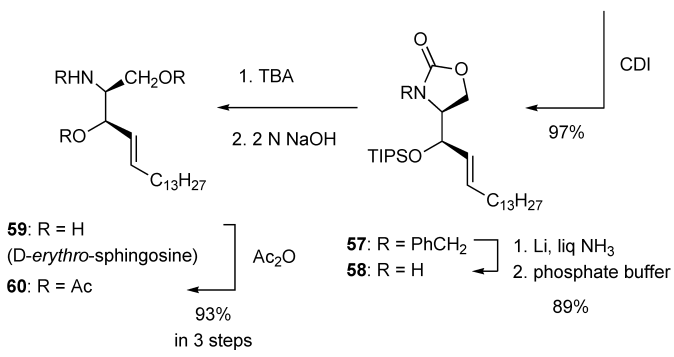

Chart 14. Enantioselective Total Synthesis of D-erythro-Sphingsine (59) from trans-(2R,3S)-3-Pentadecenylaziridine-2-carboxylate trans-45b obtained in run 2 in Table 4, was also yielded D-erythrosphingosine (59).

We succeeded in $\mathrm{C}-\mathrm{C}$ bond formation when electron-rich aromatics were used as carbon nucleophiles in the ring-opening reaction of unactivated 3-arylaziridine-2-carboxylates. ${ }^{23}$ ) An alternative ring-opening reaction with a nitrile reagent as a diverse aliphatic carbon nucleophile was examined, and diethylaluminum cyanide $\left(\mathrm{Et}_{2} \mathrm{AlCN}\right)$ was confirmed to serve as the nucleophile after several trials. ${ }^{24)}$ Preparation of the 4cyanoisoquinoline skeleton $\mathbf{6 6}$ from aziridine derivative $\mathbf{6 1}$ is shown, even as its racemic application, in Chart 15. Reaction of the $N$-(2-silyloxyethyl)guanidinium bromide $\mathbf{6 1}$ with piperonal afforded trans-aziridine-2-carboxylate $\mathbf{6 2}$ in $89 \%$ yield. After deprotection of the silyl group, followed by conversion to bromide 64, treatment with $\mathrm{Et}_{2} \mathrm{AlCN}$ gave a nitrile-inserted amino ester $\mathbf{6 5}$, which was easily cyclized to the isoquinoline skeleton 66 under Pictet-Spengler conditions.

\section{Affinity of Bisguanidines for Proton and Metal Salts (Guanidine Function as Base Ligand)}

$o$-Phenylenebis $\left(N, N^{\prime}\right.$-dimethyl- $N, N^{\prime}$-ethylene) guanidine (bisguanidine: BG) (67) was designed as a powerful hydrogen and metal cation acceptor based on the strong basic character of the guanidinyl function. Complexation of 67 with a variety of hydrogen-donor aromatics carrying hydroxyl groups such as benzoic acid (BA), phenol, and benzyl alcohol led to the successful isolation of $1: 1$ crystalline complexes, regardless of the acidity of the hydrogen donors. ${ }^{25)}$ In the case of BA, additional $1: 2,1: 3$, and $1: 4$ BG-BA crystalline complexes were formed under rigorous conditions of molar-controlled complexation (a in Fig. 2). ${ }^{26)}$ These crystals were characterized using X-ray crystallographic analysis, indicating that nonbonded interactions in addition to $\mathrm{N}-\mathrm{H}$ hydrogen bonding play an important role during the crystallization process. Mutual interconversion among the BG-BA crystalline complexes was achieved by the addition of either component to the original complex. Thus BG was found to act as a powerful hydrogen acceptor in hydrogen bond-based complexation with hydrogen-donor aromatics. We also observed that the corresponding $N$-ethyl BG analogue 68 could coordinate with a variety of metal salts such as cobalt chloride $\left(\mathrm{CoCl}_{2}\right)$ and zinc chloride $\left(\mathrm{ZnCl}_{2}\right)$ under aqueous condi-
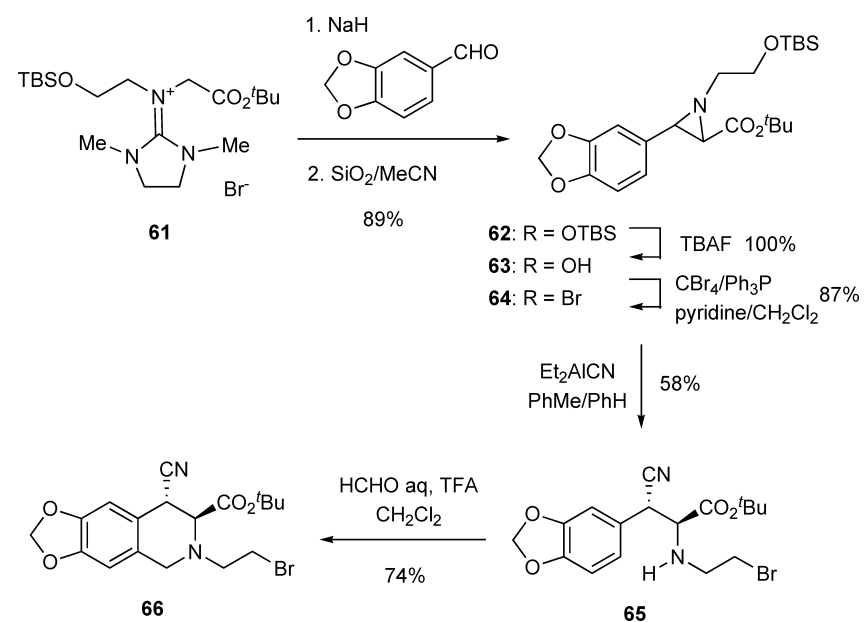

65

Chart 15. Preparation of 4-Cyanoisoquinoline Skeleton 66 as an Application of the Ring-Opening Reaction with $\mathrm{Et}_{2} \mathrm{AlCN}$ 
(a)

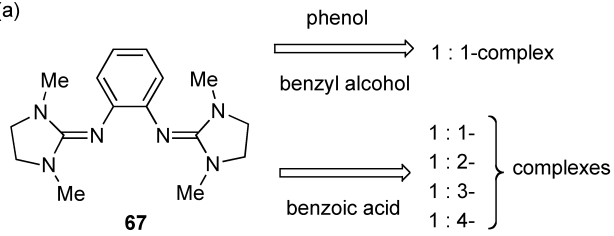

(b)

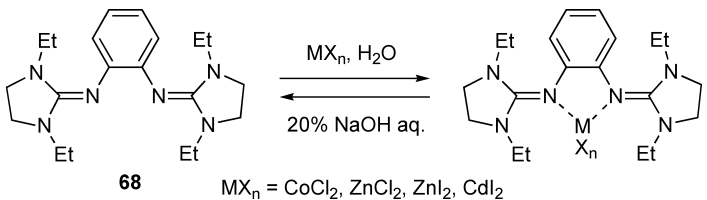

Fig. 2. Complexability of BGs: (a) with Protic Compounds; (b) with Metal Salts

tions to form a crystalline complex in some cases, which was reversible to the starting $\mathbf{6 8}$ by treatment with base (b in Fig. 2). ${ }^{27)}$

On the other hand, contamination of drinking water by arsenic is a very serious problem in the Asian region, especially in Bangladesh, and the toxic source is inorganic acid such as arsenic acid $\left(\mathrm{H}_{3} \mathrm{AsO}_{4}\right)$. The potential of BG 67 to form complexes with hydroxyl-containing organic compounds, even with neutral benzyl alcohol, led us to examine its affinity for $\mathrm{H}_{3} \mathrm{AsO}_{4}$ as a preliminary step in the removal of arsenic from polluted water. ${ }^{28)}$ We focused on: 1) the role of BGs as new Brønsted base ligands for $\mathrm{H}_{3} \mathrm{AsO}_{4}$ and phosphoric acid $\left(\mathrm{H}_{3} \mathrm{PO}_{4}\right)$, belonging to the same class-15 group; 2) the preparation of Merrifield and Hypogel resin-anchored BGs as the corresponding polymer-supported (PS) host ligands; and 3) the possibility of the immobilized BGs as potential solid-type scavengers for toxic metals and $\mathrm{H}_{3} \mathrm{AsO}_{4}$.

The expected complexation of $\mathrm{BG} 67$ with $\mathrm{H}_{3} \mathrm{AsO}_{4}$ and $\mathrm{H}_{3} \mathrm{PO}_{4}$ allowed us to prepare PS-BG derivatives. PS-BGs with a propyloxy spacer between the benzene nucleus and resin unit were designed, and cyclic and acyclic BG cores $\mathbf{8 0}$ and $\mathbf{8 1}$ were prepared, as shown in Chart 16. Successive treatment of 1,2-diaminobenzene (69) with acetylation, bromination, and alkaline hydrolysis afforded 4-bromo-1,2diaminobenzene (71) in overall $60 \%$ yield. Guanidinylation of the brominated product 71 with DMC (1) or 2-chloro1,1,3,3-tetramethylamidinium chloride (TMAC) in the presence of triethylamine smoothly gave the cyclic and acyclic guanidine analogues $\mathbf{7 2}$ and 73, respectively, in high yields. Substitution of the bromine atom with a formyl function through the bromine-lithium exchange reaction, followed by the Horner-Emmons-Wadsworth reaction, yielded 3-(3,4bisguanidinylphenyl)acrylates 76 and 77, which were subjected to catalytic hydrogenation to afford the corresponding dihydro derivatives 78 and 79. Reduction with a solution of Red-Al in THF at $-18{ }^{\circ} \mathrm{C}$ in the presence of a catalytic amount of 18-crown-6 produced BG cores $\mathbf{8 0}$ and $\mathbf{8 1}$ in $c a$. $80 \%$ yields which were sensitive to the reaction conditions (the hydride reagent should be diluted with the solvent when added) and were converted to PS-BGs by treatment with Merrifield resin and Hypogel according to the conventional method.

Evaluation of the complexability of the Merrifield and the

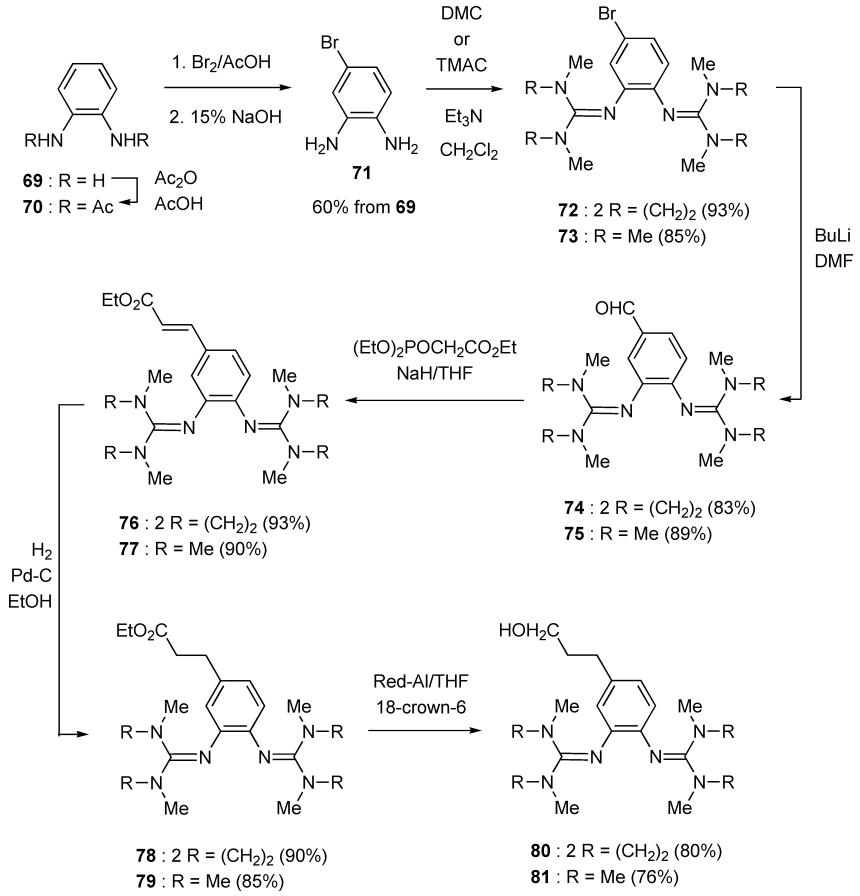

Chart 16. Preparation of BG Cores $\mathbf{8 0}$ and $\mathbf{8 1}$ for Immobilization

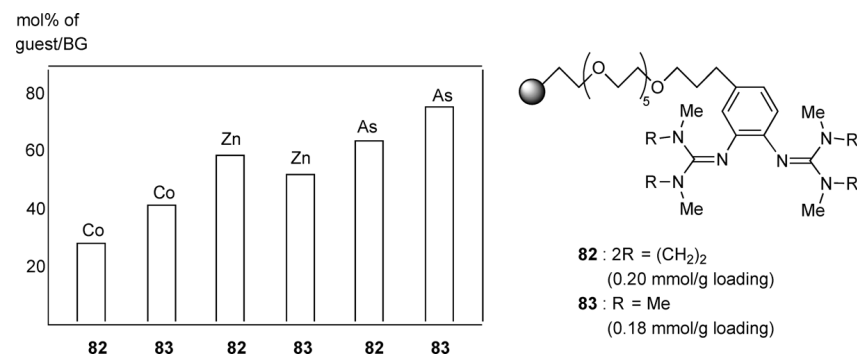

Fig. 3. Affinity of Hypogel Resin-Anchored BGs 82 and 83 for $\mathrm{CoCl}_{2}$, $\mathrm{ZnCl}_{2}$, and $\mathrm{H}_{3} \mathrm{AsO}_{4}$

Hypogel resin-anchored cyclic BGs to $\mathrm{CoCl}_{2}$ and $\mathrm{ZnCl}_{2}$ using the inductively coupled plasma mass spectrum (ICP$\mathrm{MS}$ ), in which the latter flexible PS-BG was more effective than the former rigid one, led us to examine more precisely the complexability between the Hypogel resin-anchored BGs 82 and 83 for these two metal salts and $\mathrm{H}_{3} \mathrm{AsO}_{4}$. As shown in Fig. 3, the data of the mol\% of the guest ion/PS-BG indicated that the latter acyclic PS-BG $\mathbf{8 3}$ generally acts as a more powerful ligand than the cyclic PS-BG 82.

Thus effective complexabilty of bisguanidines with either proton or metal ions in water was observed, suggesting their possible application to the removal of toxic substances from polluted water and recovery of rare metals as material sources.

\section{Desymmetrization of Bisguanidines by Alkylation (Poten- tial Chirality of Bisguanidines)}

During the above trials for complex formation between BG and BA, a chiral complex was obtained when a trace of water was incorporated in the complex, suggesting the potential chirality of BG-type compounds. ${ }^{29-31)}$ Derivatization of BG 67 to the guanidinium salts by quaternarization with alkyl halides resulted only in the formation of a monoalkyla- 

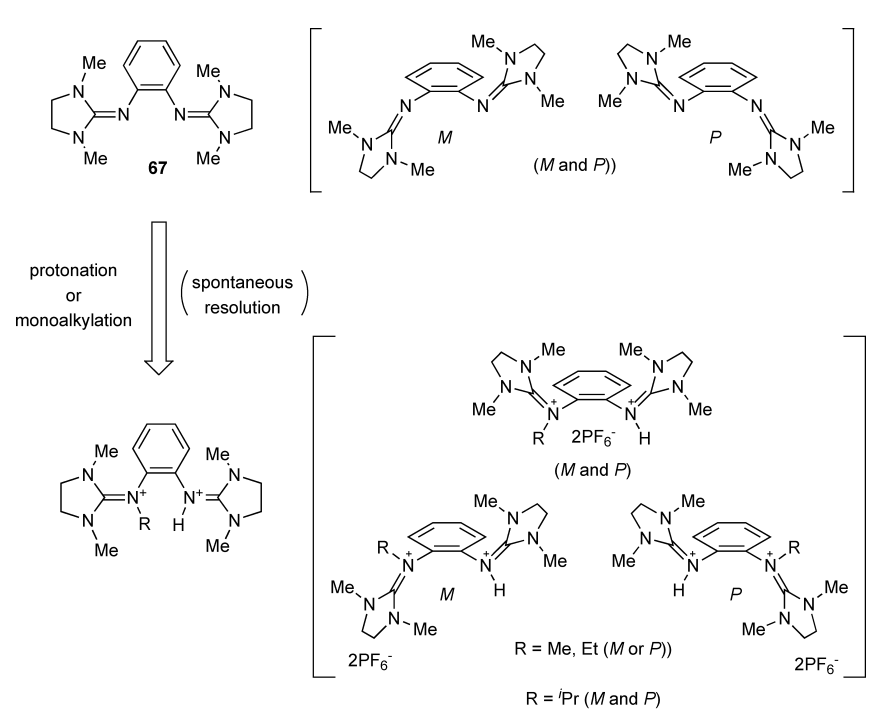

\begin{tabular}{ccccc}
\hline $\mathrm{R}$ & configuration & crystal system & space group & chirality \\
\hline- & trans & monoclinic & $P 2_{1} / \mathrm{c}$ & achiral \\
$\mathrm{H}$ & cis & monoclinic & $P 2_{1} / \mathrm{n}$ & achiral \\
$\mathrm{Me}$ & trans & orthorhombic & $P 2_{1} 2_{1} 2_{1}$ & chiral \\
$\mathrm{Et}$ & trans & orthorhombic & $P 2_{1} 2_{1} 2_{1}$ & chiral \\
$\mathrm{IPr}$ & trans & monoclinic & $P 2_{1} / \mathrm{c}$ & achiral
\end{tabular}

Fig. 4. Protonation and Monoalkylation of BG 67

tion product, not a dialkylation one, in spite of the use of a large excess amount of reagent. ${ }^{29)}$ In these derivatization experiments, although achiral crystals were produced in the cases of protonation and isopropylation, the alkylated derivatives undergo spontaneous asymmetric crystallization into a chiral form with the chiral space group $P 2_{1} 2_{1} 2_{1}$ in methylation and ethylation (Fig. 4). The absolute configurations of the chiral crystals were determined in X-ray crystallography and correlated with circular dichroism spectra recorded in the solid state. The crystallization of these compounds could be rigidly controlled with the use of a seed crystal with the desired chirality.

These findings indicate that BGs have a potential chiral character due to the plane asymmetry. We are currently investigating ways to retain the chirality of these crystals on dissolution in a solvent, as this is important in molecular recognition and asymmetric synthesis applications.

\section{Concluding Remarks}

The origin of our guanidine chemistry studies is the high reactivity of nontoxic DMC, which serves not only as a strong dehydrating agent but also as a versatile reagent in chlorination, oxidation, reduction, and rearrangements, with amines to provide modified guanidine cores with chirality easily. Examinations of them as potential chiral catalysts or auxiliaries in asymmetric synthesis, as expected, showed reasonable asymmetric inductions, in particular, the Michael reaction of diphenyliminoacetate. Reaction of guanidinium salts with aryl or unsaturated aldehydes in the presence of base led to unique aziridine formation applicable to asymmetric synthesis by the introduction of chirality to guanidinium templates. The generation of guanidinium ylides during the reaction is assumed, and a reasonable mechanism for this aziridination reaction is proposed. Guanidinium ylide chemistry newly offers different, interesting reactivity of carbonyl compounds comparable with those of known phosphorus and sulfur ylides. Furthermore, successful ring-opening reactions of aziridine products formed in this aziridination reaction with a variety of nucleophiles providing various amino acid derivatives newly revealed the synthetic utility of unactivated aziridine-2-carboxylates. The coordination ability of PS-BGs with metal salts and $\mathrm{H}_{3} \mathrm{AsO}_{4}$ in aqueous media suggested that these Brønsted base ligands could serve as effective, useful recyclable scavengers for the removal of toxic components from polluted water. In addition, spontaneous chiral crystallization in methylation and ethylation suggests the potential chiral character of BG due to the plane asymmetry. Thus our guanidine chemistry studies targeted not only synthetic organic chemistry but also environmental organic chemistry. Further development of guanidine chemistry is in progress in our laboratory.

Acknowledgments I thank the nice laboratory members of Department of Medicinal Organic Chemistry, Graduate School of Pharmaceutical Sciences, Chiba University, for their hard work on these studies, especially Dr. Toshio Isobe (Shiratori Pharmaceutical Co., Ltd.) for his great contribution to DMC chemistry leading to the elucidation of guanidine chemistry. These studies were partly supported by a Grant-in-Aid for Scientific Research from the Ministry of Education, Culture, Sports, Science and Technology, Japan. In addition, I thank the late Professor Hisashi Ishii for his enthusiastic supervision of my fundamental research careers.

\section{References}

1) Isobe T., Ishikawa T., J. Org. Chem., 64, 5832-5835 (1999).

2) Isobe T., Ishikawa T., J. Org. Chem., 64, 6984-6988 (1999).

3) Isobe T., Ishikawa T., J. Org. Chem., 64, 6989-6992 (1999).

4) Isobe T., Fukuda K., Tokunaga T., Ishikawa T., J. Org. Chem., 65, 7770-7773 (2000).

5) Isobe T., Fukuda K., Tokunaga T., Seki H., Yamaguchi K., Ishikawa T., J. Org. Chem., 65, 7774-7778 (2000).

6) Isobe T., Fukuda K., Yamaguchi K., Seki H., Tokunaga T., Ishikawa T., J. Org. Chem., 65, 7779-7785 (2000).

7) Ishikawa T., Araki Y., Kumamoto T., Seki H., Fukuda K., Isobe T., Chem. Commun., 2001, 245-246 (2001).

8) Kumamoto T., Ebine K., Endo M., Araki Y., Fushimi Y., Miyamoto I., Ishikawa T., Isobe T., Fukuda K., Heterocycles, 66, 347-359 (2005).

9) Kitani Y., Kumamoto T., Isobe T., Fukuda K., Ishikawa T., Adv. Synth. Cat., 347, 1653-1658 (2005).

10) Wannaporn D., Ishikawa T., Mol. Div., 9, 321-331 (2005).

11) Ryoda A., Yajima N., Haga T., Kumamoto T., Nakanishi W., Kawahata M., Yamaguchi K., Ishikawa T., J. Org. Chem., 73, 133-141 (2008).

12) Saito N., Ryoda A., Nakanishi W., Kumamoto T., Ishikawa T., Eur. $J$. Org. Chem., 73, 2759-2766 (2008).

13) Zhang G., Kumamoto T., Heima T., Ishikawa T., Tetrahedron Lett., 51, 3927 -3930 (2010).

14) Isobe T., Fukuda K., Ishikawa T., Tetrahedron: Asymmetry, 9, 17291735 (1998).

15) Isobe T., Fukuda K., Araki Y., Ishikawa T., Chem. Commun., 2001, $243-244$ (2001).

16) Ishikawa T., Isobe T., Chem. Eur. J., 8, 552-557 (2002).

17) Ishikawa T., Isobe T., Synth. Org. Chem. Jpn., 61, 60-68 (2003).

18) Ishikawa T., Kumamoto T., Synthesis, 2006, 737-752 (2006).

19) Hada K., Watanabe T., Isobe T., Ishikawa T., J. Am. Chem. Soc., 123, 7705-7706 (2001) [C\&EN, August 13 (2001)].

20) Wannaporn D., Ishikawa T., J. Org. Chem., 70, 9399-9406 (2005).

21) Haga T., Ishikawa T., Tetrahedron, 61, 2857-2869 (2005).

22) Wannaporn D., Ishikawa T., Kawahata M., Yamaguchi K., J. Org. Chem., 71, 6600-6603 (2006).

23) Manaka T., Nagayama S.-I., Wannaporn D., Yajima N., Kumamoto T., Watanabe T., Ishikawa T., Kawahata M., Yamaguchi K., Helv. Chim. Acta, 90, 128-142 (2007).

24) Hayashi Y., Kumamoto T., Nakanishi W., Kawahata M., Yamaguchi K., Ishikawa T., Tetrahedron, 66, 3836-3841 (2010).

25) Kawahata M., Yamaguchi K., Ishikawa T., Cryst. Grow Design, 5, 
373-377 (2005)

26) Kawahata M., Shikii K., Seki H., Ishikawa T., Yamaguchi K., Chem. Pharm. Bull., 54, 147-148 (2006).

27) Suda K., Saito N., Kumamoto T., Nakanishi W., Kawahata M., Yamaguchi K., Ogura Y., Suzuki K. T., Ishikawa T., Heterocycles, 77, $375-387$ (2009).

28) Ito T., Suda K., Kumamoto T., Nakanishi W., Watanabe T., Ishikawa T., Seki H., Kawahata M., Yamaguchi K., Ogura Y., Suzuki K. T., Mol.
Div., 14, 131-145 (2010).

29) Nakanishi W., Ogino A., Kawahata M., Yamaguchi K., Ishikawa T., Tetrahedron Lett., 48, 8526-8530 (2007).

30) Margetic D., Nakanishi W., Kumamoto T., Ishikawa T., Heterocycles, 71, 2639-2658 (2007).

31) Nakanishi W., Ishikawa T., Margetic D., Bull. Chem. Soc. Jpn., 80, 1187-1193 (2007) 